\title{
Plasma midregional proadrenomedullin in newborn infants: impact of prematurity and perinatal infection
}

\author{
Deborah Admaty' , Jörg Benzing², Tilo Burkhardt" ${ }^{3}$, Olav Lapaire4, Lukas Hegi², Gabor Szinnai ${ }^{5}$, Nils G. Morgenthaler ${ }^{6}$,
} Hans Ulrich Bucher', Christoph Bührer ${ }^{7}$ and Sven Wellmann ${ }^{1,2}$

INTRODUCTION: Adrenomedullin (ADM) is one of the strongest endogenous vasodilating hormones. Its stable by-product midregional-proADM (MR-proADM) is an established indicator of systemic infection and cardiovascular compromise in adult patients.

METHODS: A prospective cross-sectional study was performed to investigate the perinatal factors affecting MR-proADM plasma concentrations in 328 newborn infants with a gestational age (GA) between 24 and 41 wk.

RESULTS: Blood samples were obtained in 270 infants from umbilical veins (with additional 108 paired samples from umbilical arteries), and at 2-3 d of life in 183 infants. Paired venous and arterial umbilical cord MR-proADM concentrations were closely related (Spearman's rank order correlation coefficient $\left.\left(R_{s}\right)=0.825, P<0.001\right)$. MR-proADM concentrations at birth and at $2-3 \mathrm{~d}$ were inversely related to $\mathrm{GA}\left(R_{\mathrm{s}}=-0.403\right.$ and $R_{\mathrm{s}}=-0.541$, respectively) and birth weight (BW; $R_{\mathrm{s}}=-0.421$ and $R_{\mathrm{s}}=-0.530$, respectively; all $\left.P<0.001\right)$. On stepwise regression analysis, clinical chorioamnionitis and umbilical arterial blood base excess retained a significant impact on MR-proADM cord venous blood concentrations. At 2-3 d of life, histologic chorioamnionitis and GA at delivery were significantly associated with MR-proADM levels.

DISCUSSION: As compared with adults, MR-proADM concentrations are elevated in neonates, especially those born very preterm. Immaturity and infection, which both feature low systemic vascular resistance, are related to increased MR-proADM concentrations.

$\mathbf{T}$ he vasoactive peptide adrenomedullin (ADM) is produced by vascular endothelial cells, epithelial cells of various origins, and some other tissue-specific cell types $(1,2)$. ADM is involved in a variety of processes, including embryogenesis, normal and malignant growth, inflammation, and immunity $(3,4)$. Plasma concentrations of $\mathrm{ADM}$ and its stable by-product, midregionalproADM (MR-proADM), are increased in various disease processes, such as arterial hypertension, heart failure, acute coronary syndromes, renal failure, and septic shock $(3,5,6)$.
In infants, various perinatal stressors are known to increase $\mathrm{ADM}$ concentrations in umbilical cord blood or fetal membrane samples, including vaginal delivery, low umbilical cord $\mathrm{pH}$ (7), and preeclampsia (8). In two studies, it has been demonstrated that intrauterine growth restriction in response to reduced uteroplacental blood flow increases ADM concentrations in umbilical cord blood at birth $(9,10)$, whereas another study reported unchanged ADM concentrations (11). This discrepancy might be explained by different study settings and the fact that reliable quantification of ADM concentrations is hampered by its short half-life and the existence of a binding protein (12). ADM synthesis, as assessed by mRNA and protein levels, was found to be unchanged or even decreased in the placentas of women with preeclampsia (13-16). Preterm infants and infants with asphyxia who developed intraventricular hemorrhage also showed higher plasma ADM concentrations in the first days of life $(17,18)$.

Recently, reference values for MR-proADM venous cord blood concentrations have been established for healthy, term newborn infants ( $n=194$, reference range: $0.55-1.38 \mathrm{nmol} / \mathrm{l}$; ref. 19). Infants with risk factors for early-onset sepsis (EOS; $n=73)$, including intrapartum maternal fever $(n=5)$, maternal carriers of group B streptococci $(n=35)$, preterm spontaneous delivery $(n=6)$, and prolonged rupture of membranes $(n=27)$, had similar values, but no reference was made to previously identified stressors, including delivery mode, low umbilical cord $\mathrm{pH}$, or intrauterine growth restriction (19).

In this cross-sectional prospective study, we measured MR-proADM levels in plasma samples of 328 infants born between 24 and $41 \mathrm{wk}$ gestational age (GA). Samples were obtained from venous and arterial umbilical cord blood, and repeat samples were drawn at 2-3 d of life concomitant with metabolic screening. The findings were related to indexes of pregnancy, parturition, placental histology, and postnatal course.

\section{RESULTS}

Patients

Patient characteristics are given in Table 1. 
Table 1. Clinical details of infants grouped according to gestational age

\begin{tabular}{|c|c|c|c|c|c|c|c|c|}
\hline \multirow{3}{*}{ Maternal characteristics } & \multirow[b]{2}{*}{$n$} & \multicolumn{2}{|c|}{ GA 24-31 wk } & \multicolumn{2}{|c|}{ GA 32-36 wk } & \multicolumn{2}{|c|}{ GA 37-41 wk } & \multirow[b]{2}{*}{$P$} \\
\hline & & \multirow[t]{2}{*}{$\begin{array}{l}M(\mathrm{P} 10 / \mathrm{P} 90) \\
\quad \text { or } n(\%)\end{array}$} & $N$ & \multirow[t]{2}{*}{$\begin{array}{l}M(\mathrm{P} 10 / \mathrm{P} 90) \\
\text { or } n(\%)\end{array}$} & $n$ & \multirow[t]{2}{*}{$\begin{array}{c}M(\mathrm{P} 10 / \mathrm{P} 90) \\
\text { or } n(\%)\end{array}$} & $N$ & \\
\hline & & & & & & & & \\
\hline & 328 & $32(26 / 39)$ & 86 & $34(26 / 40)$ & 99 & $32(24 / 39)$ & 143 & NS \\
\hline BMI before pregnancy & 284 & $23(19 / 28)$ & 73 & $22(19 / 29)$ & 91 & $23(20 / 28)$ & 120 & NS \\
\hline Preeclampsia & 328 & $26(30)$ & 86 & $24(24)$ & 99 & $3(2)$ & 143 & $<0.001$ \\
\hline PPROM & 328 & $32(37)$ & 86 & $39(39)$ & 99 & $15(11)$ & 141 & $<0.001$ \\
\hline Antenatal steroids, full course & 328 & $75(87)$ & 86 & $35(35)$ & 99 & $2(1)$ & 143 & $<0.001$ \\
\hline Antenatal steroids, any & 328 & $84(98)$ & 86 & $46(46)$ & 99 & $0(0)$ & 143 & $<0.001$ \\
\hline \multicolumn{9}{|l|}{ At delivery } \\
\hline CRP (mg/l) & 191 & $6(2 / 36)$ & 79 & $4(2 / 17)$ & 87 & $3(1 / 20)$ & 25 & $<0.01$ \\
\hline Histologic chorioamnionitis & 168 & $16(19)$ & 85 & $3(5)$ & 65 & $0(0)$ & 18 & $<0.01$ \\
\hline Maternal reaction only & 168 & $10(12)$ & 85 & $2(3)$ & 65 & $0(0)$ & 18 & $<0.05$ \\
\hline Maternal + fetal reaction & 168 & $6(7)$ & 85 & $1(2)$ & 65 & $0(0)$ & 18 & $<0.05$ \\
\hline Compromised perfusion & 168 & $35(41)$ & 85 & $26(40)$ & 65 & $9(50)$ & 18 & NS \\
\hline \multicolumn{9}{|l|}{ Fetal characteristics } \\
\hline Small for gestational age $(<P 5)$ & 328 & $14(16)$ & 86 & $21(21)$ & 99 & $14(10)$ & 143 & NS \\
\hline Suspect CTG just before birth & 328 & $30(35)$ & 86 & $27(27)$ & 99 & $48(34)$ & 143 & NS \\
\hline \multicolumn{9}{|l|}{ Infants } \\
\hline $\operatorname{Sex}(m / f)$ & 328 & $44 / 42$ & 86 & $57 / 42$ & 99 & $84 / 59$ & 143 & NS \\
\hline \multicolumn{9}{|l|}{ At birth } \\
\hline Base deficiency $(\mathrm{mmol} / \mathrm{l})$ arterial & 241 & $-4.0(-0.8 /-8.6)$ & 25 & $-2.4(-0.5 /-8.5)$ & 87 & $-3.2(-0.6 /-7.3)$ & 129 & NS \\
\hline Apgar 5 min & 328 & $7(3 / 9)$ & 86 & $9(7 / 10)$ & 99 & $9(9 / 10)$ & 143 & $<0.001$ \\
\hline Apgar $10 \mathrm{~min}$ & 328 & $8(6 / 9)$ & 86 & $9(8 / 10)$ & 99 & $9(9 / 10)$ & 143 & $<0.001$ \\
\hline \multicolumn{9}{|l|}{ Postnatal course until 2-3d } \\
\hline Endotracheal ventilation & 328 & $33(38)$ & 86 & $1(1)$ & 99 & $0(0)$ & 143 & $<0.001$ \\
\hline Any positive pressure support & 328 & $65(76)$ & 86 & $16(16)$ & 99 & $0(0)$ & 143 & $<0.001$ \\
\hline Early-onset sepsis proven & 328 & $5(6)$ & 86 & $2(2)$ & 99 & $0(0)$ & 143 & $<0.01$ \\
\hline Patent ductus arteriosus treated & 243 & $27(31)$ & 86 & $2(2)$ & 99 & $0(0)$ & 58 & $<0.001$ \\
\hline Intraventricular hemorrhage, $>$ I & 136 & $7(8)$ & 86 & $0(0)$ & 50 & $0(0)$ & 0 & NS \\
\hline \multicolumn{9}{|l|}{ Postnatal course until discharge } \\
\hline Necrotizing enterocolitis, >lla & 328 & $1(1)$ & 86 & $0(0)$ & 99 & $0(0)$ & 143 & NS \\
\hline Retinopathy of prematurity, $>1$ & 84 & $3(3)$ & 84 & $0(0)$ & 0 & $0(0)$ & 0 & - \\
\hline Moderate/severe BPD & 328 & $6(7)$ & 86 & $0(0)$ & 99 & $0(0)$ & 143 & $<0.001$ \\
\hline Death & 328 & $8(9)$ & 86 & $1(1)$ & 99 & $0(0)$ & 143 & $<0.001$ \\
\hline \multicolumn{9}{|l|}{ MR-proADM (nmol/l) } \\
\hline At birth, venous cord blood & 270 & $1.4(0.9 / 2.2)$ & 74 & $1.1(0.8 / 1.8)$ & 82 & $1.0(1.0 / 1.4)$ & 114 & $<0.001$ \\
\hline At $2-3$ d of life & 183 & $1.4(0.9 / 2.3)$ & 57 & $0.9(0.4 / 1.4)$ & 47 & $0.9(0.7 / 1.0)$ & 79 & $<0.001$ \\
\hline
\end{tabular}

Characteristics were compared using Mann-Whitney $U$ test or Fisher's exact test as appropriate. $P>0.05$ was considered nonsignificant.

BMI, body mass index; BPD, bronchopulmonary dysplasia, oxygen at 36 wk postmenstrual age; CRP, C-reactive protein; CTG, cardiotocogram; GA, gestational age; $M$, median; m/f, male/ female; MR-proADM, midregional proadrenomedullin; NS, not significant; P, percentile; PPROM, prolonged premature rupture of membranes. 
MR-proADM Concentrations in Paired Arterial and Venous Umbilical Cord Plasma Samples

Paired umbilical cord arterial and venous plasma MR-proADM concentrations were closely related $\left(R_{\mathrm{s}}=0.825, P<0.001, n=\right.$ 108; Figure 1). Overall, MR-proADM concentrations were slightly higher in venous, as compared with arterial samples (mean $(M)=1.03$ vs. $1.01 \mathrm{nmol} / 1, P<0.05)$. In $76(70.4 \%)$ of 108 pairs, MR-proADM concentrations were higher in venous than arterial samples, whereas in the remaining 32 (29.6\%) cases umbilical arterial MR-proADM exceeded venous MR-proADM. No differences in clinical parameters were found when comparing the two groups.

Relationship Between GA and MR-proADM Plasma Concentrations at Birth and at 2-3 d

Birth weight (BW) and GA at birth were inversely related to MR-proADM concentrations in venous umbilical cord plasma $\left(R_{\mathrm{s}}=-0.421, R_{\mathrm{s}}=-0.403\right.$, respectively, both $P<0.001, n=270$; Figure 2a) and to MR-proADM concentrations in plasma from $2-3 \mathrm{~d}$ of life $\left(R_{\mathrm{s}}=-0.530, R_{\mathrm{s}}=-0.541\right.$, respectively, both $P<0.001, n=183$; Figure 2b).

Paired plasma MR-proADM concentrations at birth and 2-3 $\mathrm{d}$ of life were directly correlated $\left(R_{\mathrm{s}}=0.367, P<0.001, n=125\right)$. MR-proADM concentrations decreased in term (GA 37-41 wk) and moderately preterm infants (GA $32-36 \mathrm{wk}$ ) but not in very preterm infants (GA 24-31 wk), as shown in Figure 3.

Impact of Labor on Cord Blood MR-proADM Plasma Concentrations

Term infants born by elective cesarean section $(n=55)$ had significantly lower MR-proADM concentrations in cord blood than infants born by vaginal delivery $(n=41)$ or secondary cesarean section (after the onset of labor, $n=18 ; M=0.93$ vs. 1.15 and $1.18 \mathrm{nmol} / 1$, respectively, $P<0.001)$. In preterm infants, there was no impact of delivery mode on MR-proADM concentrations.

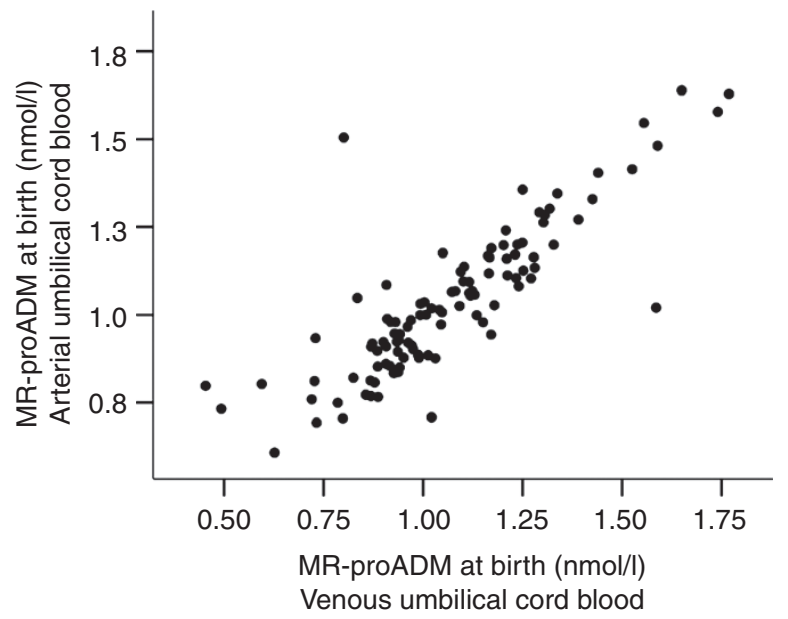

Figure 1. Scatter plots of paired arterial and venous umbilical cord plasma MR-proADM concentrations at birth. Spearman's rank order correlation coefficient $(R)=0.848, P<0.001, n=108$. MR-proADM, midregional proadrenomedullin.
Arterial umbilical cord blood $\mathrm{pH}$ did not correlate with MR-proADM concentrations at birth among all infants $(n=270)$ or infants stratified by GA. Arterial umbilical cord blood base deficit, however, was inversely related to MR-proADM concentrations at birth in the total cohort $\left(R_{\mathrm{s}}=-0.414, P<0.001, n=196\right)$ and subsets defined by GA (term $(n=103): R_{\mathrm{s}}=-0.514, P<0.001$; moderate preterm: $(n=70) R_{\mathrm{s}}=-0.326, P<0.01$; very preterm: $(n=23)$ $\left.R_{\mathrm{s}}=-0.414, P<0.05\right)$. Five- and $10-\mathrm{min}$ Apgar scores were related to MR-proADM concentrations in cord blood for preterm infants only $\left(n=74 ; R_{\mathrm{s}}=-0.412, P<0.001\right.$ and $R_{\mathrm{s}}=$ $-0.266, P<0.5$, respectively).

Increased MR-proADM Plasma Concentrations at Birth and With Perinatal Infection

Variables associated with perinatal infection (maternal C-reactive protein and leukocyte count immediately before the onset of parturition, intrapartum fever $\geq 38^{\circ} \mathrm{C}$, histological signs of chorioamnionitis, and bloodstream-proven EOS) were each significantly related to MR-proADM plasma concentrations in venous umbilical cord blood at birth: the continuous
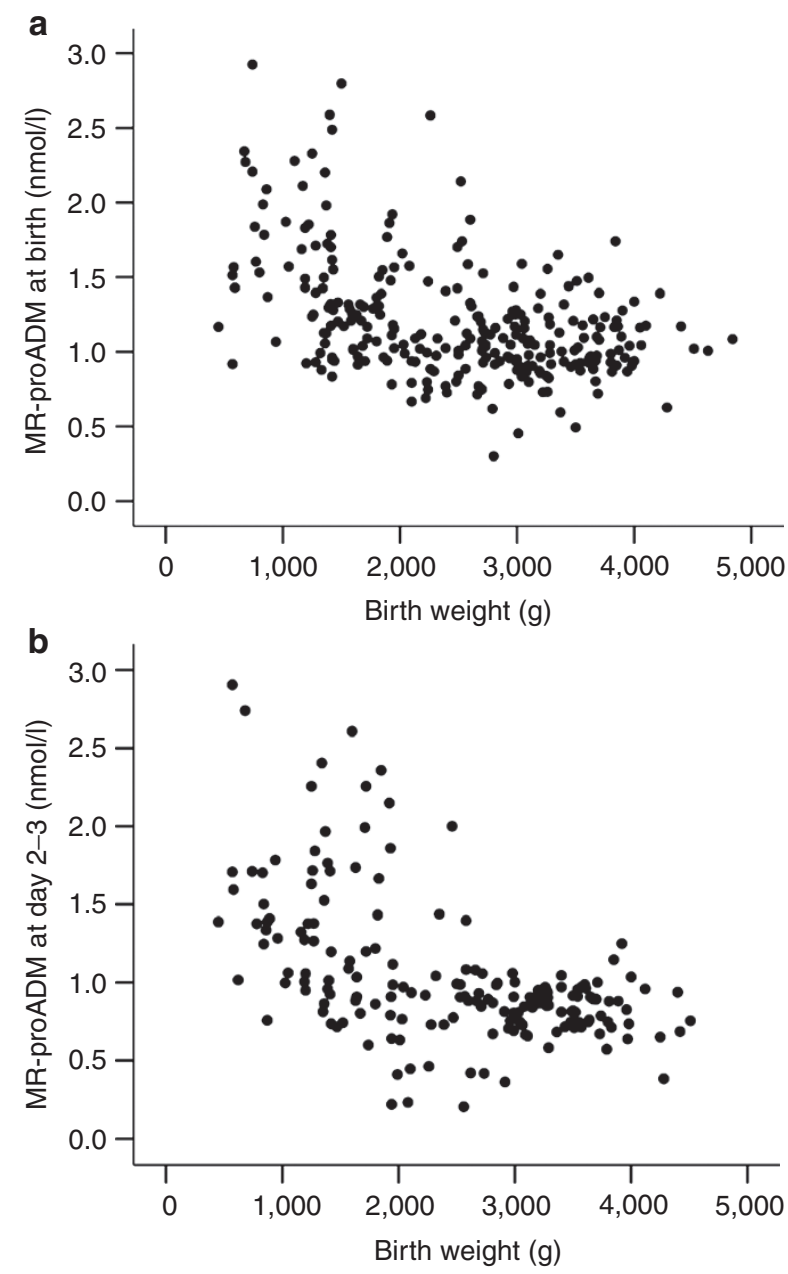

Figure 2. Relationships between birth weight and MR-proADM concentrations at (a) birth in venous umbilical cord blood $\left(R_{\mathrm{s}}=-0.421\right.$, $P<0.001, n=270)$ or at (b) $2-3 \mathrm{~d}$ of life $\left(R_{\mathrm{s}}=-0.531, P<0.001, n=183\right)$. MR-proADM, midregional proadrenomedullin. 


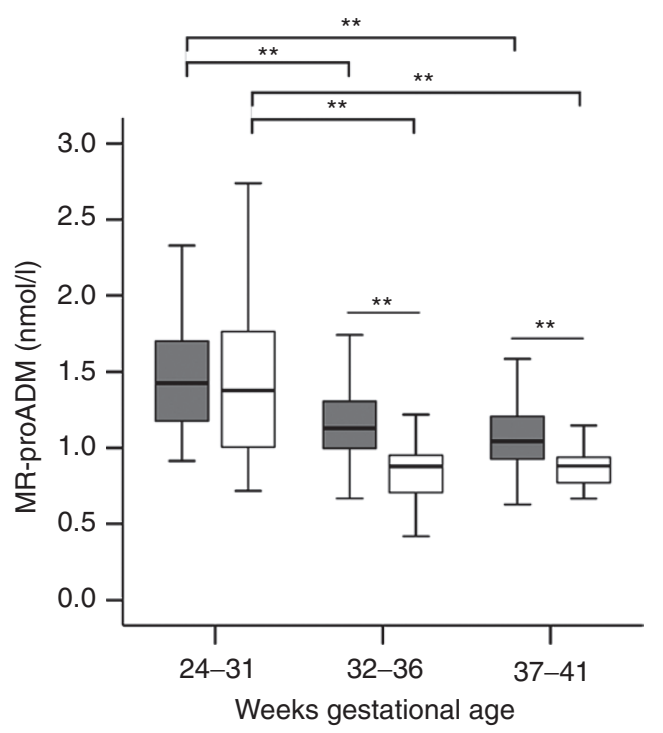

Figure 3. MR-proADM concentrations in paired samples of venous umbilical cord blood at birth (gray boxes) and at 2-3 d (white boxes) of life in infants with gestational age $<32 \mathrm{wk}(n=45), 32-36 \mathrm{wk}(n=30)$, or 37-41 wk $(n=50)$. MR-proADM concentrations are presented as box (interquartile range) and whisker (5-95\% range) plots. Levels of significance between various groups were calculated by Mann-Whitney $U$ test as indicated $(* * 0.001)$. MR-proADM, midregional proadrenomedullin.

maternal parameters C-reactive protein and leukocyte count correlated directly with MR-proADM at birth, $R_{\mathrm{s}}=0.258(n=$ $162)$ and $0.270(n=197)$, respectively (both $P<0.001)$. In the total group of infants with venous umbilical cord blood samples at birth $(n=270)$, MR-proADM concentrations at birth were significantly elevated in the presence of any of the following variables: clinical chorioamnionitis $(M=1.60 \mathrm{nmol} / \mathrm{l}$, range $0.8-2.59, n=23)$ vs. $M=1.09 \mathrm{nmol} / \mathrm{l}$, range $0.3-3.42, n=247$, $P<0.001)$; histologic chorioamnionitis $(M=1.74 \mathrm{nmol} / \mathrm{l}$, range $0.93-2.59, n=15$ vs. $M=1.18 \mathrm{nmol} / \mathrm{l}$, range $0.62-3.42, n=128$, $P<0.001)$; blood-stream proven EOS $(M=1.57 \mathrm{nmol} / \mathrm{l}$, range $1.17-1.72, n=5$ vs. $M=1.11 \mathrm{nmol} / \mathrm{l}$, range $0.3-3.42, n=265$, $P<0.001)$. Similar results were obtained when analyzing only preterm infants with venous umbilical cord blood samples at birth $(n=156)$, as shown in Figure 4.

As amnion infection is a leading cause of preterm delivery, and prematurity was inversely related to MR-proADM concentrations, we performed a stepwise multiple regression analysis, including GA at delivery, arterial umbilical cord blood $\mathrm{pH}$, arterial umbilical cord blood base excess, and clinical chorioamnionitis. Clinical chorioamnionitis $(P<0.001)$ and umbilical cord blood base excess $(P<0.001)$ retained a significant impact on MR-proADM cord blood concentrations but not $\mathrm{GA}$ at delivery or umbilical cord blood $\mathrm{pH}$.

\section{MR-proADM Plasma Concentrations at 2-3 d}

Each of the various infant variables recorded during the postnatal course was strongly related to prematurity (Table 1 ). Even within the group of extremely premature infants, all of the variables studied were significantly related to GA and BW. However, within the group of extremely premature infants,

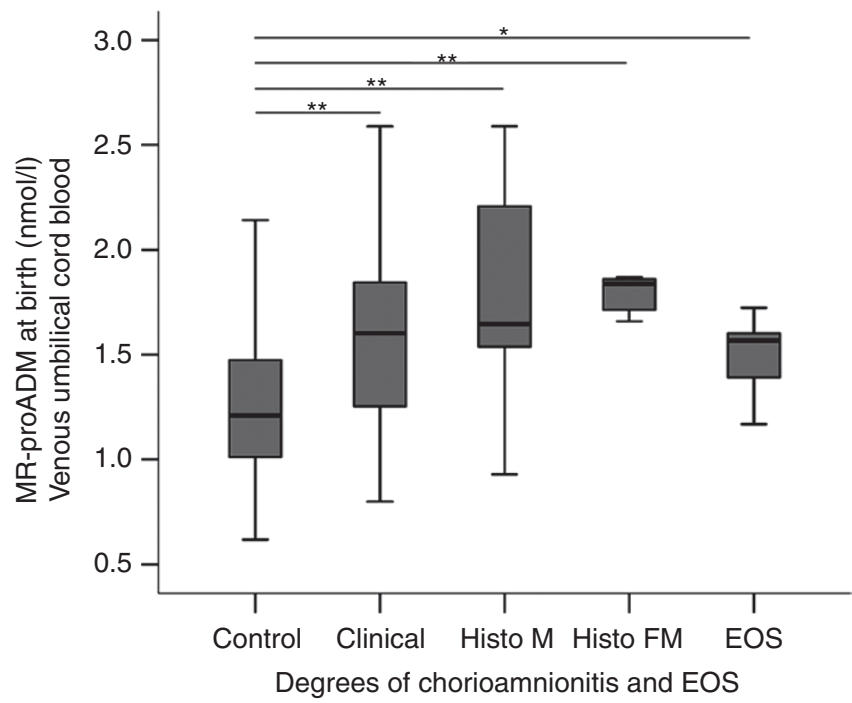

Figure 4. MR-proADM concentrations at birth were higher in preterm infants when chorioamnionitis or EOS was noted. Of 156 preterm infants with venous umbilical cord blood samples at birth, neither clinical nor histologic chorioamnionitis nor EOS was recorded in 127 subjects (Control). Clinical chorioamnionitis was present in 22 of 156 preterms (Clinical, in 12 subjects chorioamnionitis was verified by histologic examination). Histo $\mathrm{M}$ was found in 8 of 156 preterms, whereas Histo FM was found in 7 of 156 preterms. Bloodstream-proven EOS was diagnosed in 5 of 156 preterm infants. MR-proADM concentrations are presented as box (interquartile range) and whisker (5-95\% range) plots. Levels of significance between groups were calculated by Mann-Whitney $U$ test as indicated $\left({ }^{*} P<0.05\right.$; $\left.{ }^{* *} P<0.01\right)$. EOS, early-onset sepsis; Histo FM, histologic chorioamnionitis with both maternal and fetal reaction; Histo $\mathrm{M}$, histologic chorioamnionitis with only maternal reaction; MR-proADM, midregional proadrenomedullin.

MR-proADM concentrations at $2-3 \mathrm{~d}$ did not correlate with GA $\left(R_{\mathrm{s}}=-0.047, P=0.731\right)$ or $\mathrm{BW}\left(R_{\mathrm{s}}=-0.076 . P=0.473\right)$ but were significantly $(P<0.01)$ higher in infants receiving any positive pressure respiratory support (endotracheal ventilation: $M=1.5$ (range $0.74-3.8$ ) nmol/l, $n=25$; noninvasive respiratory support (nasal or pharyngeal continuous positive airway pressure: $M=1.28$ (range $0.74-2.6) \mathrm{nmol} / \mathrm{l}, n=21$ ) as compared with those without any positive pressure support $(M=$ 1.01 (range $0.72-1.86$ ) $\mathrm{nmol} / \mathrm{l}, n=11$ ). The other variables had no influence on MR-proADM concentrations at 2-3 d.

Clinical chorioamnionitis was associated with MR-proADM concentrations at $2-3 \mathrm{~d}(P=0.03)$, applying a stepwise regression model together with GA at delivery $(P=0.168)$. A further stepwise regression model was applied to test histologic chorioamnionitis and GA at delivery as potential influencing markers on MR-proADM concentrations at 2-3 d of life. The regression analyses revealed a significant impact of both histologic chorioamnionitis $(P=0.033)$ and GA at delivery $(P<0.001)$ on MR-proADM levels at 2-3 d of life.

\section{DISCUSSION}

This prospective cross-sectional study presents data concerning the concentrations of plasma MR-proADM in preterm and term infants at birth and at 2-3 d of life. This is the first study of its kind to present such data. The major findings are as follows: (i) MR-proADM plasma concentrations are closely 
related in paired arterial and venous umbilical cord samples at birth. (ii) The more preterm an infant is at birth, the higher MR-proADM plasma concentrations are at birth and 2-3 d. (iii) Irrespective of GA and BW, MR-proADM plasma concentrations are increased at birth in infants of mothers with clinical or histologic chorioamnionitis and signs of antenatal cardiovascular compromise (base deficit). (iv) At 2-3 d of life, respiratory support is associated with higher MR-proADM plasma concentrations.

The concept of replacing the cumbersome measurement of bioactive and rapidly cleared peptides by measurement of their stable, nonfunctional, and cognate peptides derived from a common precursor has greatly increased our capacity for both diagnosis and prognosis. This improvement in assay technology has implemented MR-proADM measurements in several large clinical trials in adult medicine $(20,21)$.

Although many studies have been conducted on plasma concentrations of bioactive ADM in preterm and term newborns $(7-10,17-18,22)$, just one analysis has been published thus far on MR-proADM in healthy term newborns. This analysis described values for MR-proADM in venous umbilical cord plasma (19). Term infants born without risk factors for vertically transmitted infections had slightly lower MR-proADM values $(M=0.84$, range $=0.55-1.38 \mathrm{nmol} / \mathrm{l})$ than infants with these risk factors but without signs of sepsis $(M=\sim 0.88$, range $=0.11-2.54 \mathrm{nmol} / \mathrm{l})$. These values are very similar to our data on healthy term newborns and clearly above the circulating plasma MR-proADM concentrations published for healthy adults $(M=0.33$, range $=$ $0.10-0.64 \mathrm{nmol} / \mathrm{l}$; ref. 12).

Moreover, our data unequivocally show that the more preterm an infant was born, the higher MR-proADM plasma concentrations were at birth and at 2-3 d (Figure 3). This inverse relation of MR-proADM concentrations at birth and at 2-3 d of life to GA as well as BW denotes an involvement for ADM in development, specifically the development of the cardiovascular system. ADM is a major endogenous vasodilatator (2) and blood pressure and prematurity are related in a linear fashion, i.e., the lower the GA at birth, the lower the blood pressure during the first days of life (23). An increasing body of literature has led to the present attitude of permissive hypotension in the extremely low-birth-weight infant when signs of good perfusion are present (24). We speculate that high levels of ADM in preterm infants result in low systemic vascular resistance and hence low blood pressure. With the drop in ADM, blood pressure increases during the first days unless there is diastolic run-off through a patent ductus arteriosus.

Our finding of an association of respiratory support with higher MR-proADM plasma concentrations at $2-3 \mathrm{~d}$ is supported by the data of Kamata et al., as they demonstrated elevated levels of plasma ADM in umbilical cord blood at birth and at 3 and $6 \mathrm{~d}$ of life in infants with persistent pulmonary hypertension neonatorum (25). This supports the notion that circulating ADM plays a role in regulating pulmonary vascular tone (26) and may be involved in lung differentiation and maturation (27).
Bacterial endotoxins and inflammatory cytokines induce the expression of $\operatorname{ADM}(28,29)$, and both the secretion of $\mathrm{ADM}$ and MR-proADM are increased during sepsis in adults $(30,31)$ and neonates $(32)$. These data are in line with our findings of elevated MR-proADM concentrations in cord blood at birth in infants with EOS and/or chorioamnionitis. Arterial hypotension is a common finding in sepsis and may be caused in part by elevated ADM, which, together with the ADMbinding protein, also has anti-inflammatory properties (33).

Base deficit in umbilical cord blood is a marker of chronic fetal stress and indicates increased anaerobic glycolysis, which is caused by systemic fetal hypoxia or regional hypoxia when microcirculation is impaired, e.g., in inflammation. Placental ADM mRNA and protein, as well as secreted ADM, have been shown to be increased in response to hypoxia in experimental settings (34-36). This supports our finding that chronic fetal stress is related to elevated MR-proADM plasma concentrations at birth. In contrast, arterial umbilical cord blood $\mathrm{pH}$ is a marker of acute birth stress and vaginal delivery. As compared with elective abdominal delivery, vaginal delivery elicits a dramatic surge in stress hormones in term and preterm infants as shown earlier $(37,38)$. MR-proADM concentrations at birth did not show any association with arterial umbilical cord blood $\mathrm{pH}$, and only in term infants were MR-proADM concentrations at birth slightly increased in infants born vaginally as compared with infants born by elective caesarean section $(M=1.15$ vs. $0.93 \mathrm{nmol} / \mathrm{l}$, respectively, $P<0.001)$.

In contrast to previous studies employing bioactive ADM in cord blood (8), MR-proADM concentrations were not altered in our study cohort when preeclampsia was present. It has been reported that values of bioactive ADM measured $6 \mathrm{~h}$ after birth were elevated in preterm infants developing intraventricular hemorrhage (17). Within our cohort of very preterm infants (GA 24-31 wk, $n=90$ ), eight infants developed intraventricular hemorrhage of different grades. Neither MR-proADM concentrations in cord blood at birth nor at $2-3 \mathrm{~d}$ were significantly increased in these infants.

An important limitation of this exploratory study is the small number of infants with serious morbidities, including intraventricular hemorrhage and bloodstream proven EOS. Whether or not MR-proADM may offer clinically helpful pieces of information in sick preterm infants warrants differentially designed studies focusing on this question.

In conclusion, MR-proADM concentrations at birth are associated with immaturity and inflammation. The elevated MR-proADM concentrations may be causally linked to reduced systemic vascular resistance observed in these two conditions.

\section{METHODS}

Between March 2009 and December 2010, a total of 328 infants born at the University Hospitals of Basel and Zürich were enrolled in this prospective, cross-sectional study after obtaining informed written consent from the parents. The study was approved by the institutional review boards of the University Hospital of Basel and Zürich (Ethikkommission beider Basel, EKBB 07/09, Kantonale Ethikkommission Zürich, KEK 08/09). 
Blood samples were drawn from 270 umbilical veins at the time of delivery, with an additional 108 paired samples from the umbilical artery and an additional 125 paired blood samples from postnatal day 2-3. Additional blood samples were taken at postnatal day 2-3 from 58 infants. After collecting blood in EDTA tubes, samples were stored at $4{ }^{\circ} \mathrm{C}$ until centrifugation, performed within $<8 \mathrm{~h}$. Plasma was transferred into a new EDTA tube and subsequently frozen at $-28^{\circ} \mathrm{C}$. MR-proADM (nmol/l) was measured in a single batch with BRAHMS KRYPTOR automated immunofluorescent assay (BRAHMS Biomarkers; Thermo Fisher Scientific, Hennigsdorf, Germany) (39).

Details of the pregnancy, delivery, and the infants' birth were collected from the charts. Placentas were histologically examined for signs of chorioamnionitis (maternal and fetal reaction) and altered placental perfusion capacity by the local pathologists (Rosmarie Caduff, Institute of Pathology, University Hospital Zurich; Elisabeth Bruder, Institute of Pathology, University Hospital Basel). When the various pathomorphological findings, including impaired maternal blood flow through the intervillous space (infarcts) or impaired fetal blood flow through the cord and allantoic vessels or chorionic villi (fetal thrombotic vasculopathy) were considered significant, decreased placental perfusion capacity was diagnosed (40). Prolonged premature rupture of the membranes was defined as rupture of membranes more than 18 hours before labor and before $37 \mathrm{wk}$ gestation. Data concerning the infants' health after birth were recorded by the staff on the maternity or neonatology wards. Clinical data were recorded as described previously (41) based on the standardized definitions of the Swiss Neonatal Network. Both invasive (endotracheal ventilation) and noninvasive (nasal or pharyngeal continuous positive airway pressure) ventilation was counted as positive pressure support. Management of patent ductus arteriosus, including diagnosis and treatment, was performed as previously published (42). Clinical chorioamnionitis was diagnosed in women who had three or more of the following signs: leukocytes $>20,000 / \mu \mathrm{l}, \mathrm{C}$-reactive protein $>40 \mathrm{mg} / \mathrm{dl}$, temperature $>38^{\circ} \mathrm{C}$, maternal tachycardia $>100 \mathrm{bpm}$, and fetal tachycardia $>160 \mathrm{bpm}$.

A stepwise multiple regression was applied to assess the impact of GA at delivery, arterial umbilical cord blood $\mathrm{pH}$, arterial umbilical cord blood base excess, and clinical chorioamnionitis on plasma MR-proADM alterations in venous umbilical cord blood whereas GA at delivery, clinical chorioamnionitis and histologic chorioamnionitis were investigated as variables affecting infant MR-proADM levels at 2-3 d of life.

Data were analyzed employing IBM SPSS Statistics 19.0 (SPSS, Chicago, IL) in a strictly nonparametric fashion (Mann-Whitney $U$, Kruskal-Wallis, and Fisher's exact tests). All continuous variables are reported as $M$ (range). $R_{\mathrm{s}}$ values were calculated to characterize the relationships between continuous variables. A $P$-value $<0.05$ was considered to be significant.

\section{ACKNOWLEDGMENTS}

We are grateful to Rosmarie Caduff, Institute of Pathology, University Hospital Zurich, and to Elisabeth Bruder, Institute of Pathology, University Hospital Basel, for histological examination of placentas. We thank the midwives, the neonatal intensive care unit (NICU) nurses, and the NICU doctors for their assistance during the examinations.

\section{STATEMENT OF FINANCIAL SUPPORT}

S.W. was supported by a Swiss National Science Foundation Career Award for Medical Scientists (33CM30-124101).

\section{REFERENCES}

1. Kitamura K, Kangawa K, Kawamoto M, et al. Adrenomedullin: a novel hypotensive peptide isolated from human pheochromocytoma. Biochem Biophys Res Commun 1993;192:553-60.

2. Hinson JP, Kapas S, Smith DM. Adrenomedullin, a multifunctional regulatory peptide. Endocr Rev 2000;21:138-67.

3. Beltowski J, Jamroz A. Adrenomedullin-what do we know 10 years since its discovery? Pol J Pharmacol 2004;56:5-27.

4. Li M, Yee D, Magnuson TR, Smithies O, Caron KM. Reduced maternal expression of adrenomedullin disrupts fertility, placentation, and fetal growth in mice. J Clin Invest 2006;116:2653-62.
5. Chan D, Ng LL. Biomarkers in acute myocardial infarction. BMC Med 2010;8:34.

6. Christ-Crain M, Opal SM. Clinical review: the role of biomarkers in the diagnosis and management of community-acquired pneumonia. Crit Care 2010;14:203.

7. Boldt T, Luukkainen P, Fyhrquist F, Pohjavuori M, Andersson S. Birth stress increases adrenomedullin in the newborn. Acta Paediatr 1998;87:93-4.

8. Di Iorio R, Marinoni E, Letizia C, Alò P, Villaccio B, Cosmi EV. Adrenomedullin, a new vasoactive peptide, is increased in preeclampsia. Hypertension 1998;32:758-63.

9. Di Iorio R, Marinoni E, Letizia C, Gazzolo D, Lucchini C, Cosmi EV. Adrenomedullin is increased in the fetoplacental circulation in intrauterine growth restriction with abnormal umbilical artery waveforms. Am J Obstet Gynecol 2000;182:650-4.

10. Yamashiro C, Hayashi K, Yanagihara T, Hata T. Plasma adrenomedullin levels in pregnancies with appropriate for gestational age and small for gestational age infants. J Perinat Med 2001;29:513-8.

11. Akturk A, Onal EE, Atalay Y, et al. Maternal and umbilical venous adrenomedullin and nitric oxide levels in intrauterine growth restriction. J Matern Fetal Neonatal Med 2007;20:521-5.

12. Morgenthaler NG, Struck J, Alonso C, Bergmann A. Measurement of midregional proadrenomedullin in plasma with an immunoluminometric assay. Clin Chem 2005;51:1823-9.

13. Knerr I, Dachert C, Beinder E, et al. Adrenomedullin, calcitonin generelated peptide and their receptors: evidence for a decreased placental mRNA content in preeclampsia and HELLP syndrome. Eur J Obstet Gynecol Reprod Biol 2002;101:47-53.

14. Kanenishi K, Kuwabara H, Ueno M, Sakamoto H, Hata T. Immunohistochemical adrenomedullin expression is decreased in the placenta from pregnancies with pre-eclampsia. Pathol Int 2000;50:536-40.

15. Jerat S, Morrish DW, Davidge ST, Kaufman S. Effect of adrenomedullin on placental arteries in normal and preeclamptic pregnancies. Hypertension 2001;37:227-31.

16. Al-Ghafra A, Gude NM, Brennecke SP, King RG. Increased adrenomedullin protein content and mRNA expression in human fetal membranes but not placental tissue in pre-eclampsia. Mol Hum Reprod 2006;12:181-6.

17. Gazzolo D, Marinoni E, Giovannini L, Letizia C, Serra G, Di Iorio R. Circulating adrenomedullin is increased in preterm newborns developing intraventricular hemorrhage. Pediatr Res 2001;50:544-7.

18. Di Iorio R, Marinoni E, Lituania M, et al. Adrenomedullin increases in term asphyxiated newborns developing intraventricular hemorrhage. Clin Biochem 2004;37:1112-6.

19. Miguel D, Prieto B, Costa M, Coto D, Alvarez FV. Cord blood plasma reference intervals for potential sepsis markers: pro-adrenomedullin, pro-endothelin, and pro-atrial natriuretic peptide. Clin Biochem 2011;44:337-41.

20. Maisel A, Mueller C, Nowak R, et al. Mid-region pro-hormone markers for diagnosis and prognosis in acute dyspnea: results from the $\mathrm{BACH}$ (Biomarkers in Acute Heart Failure) trial. J Am Coll Cardiol 2010;55: 2062-76.

21. Schuetz P, Wolbers M, Christ-Crain M, et al.; ProHOSP Study Group. Prohormones for prediction of adverse medical outcome in communityacquired pneumonia and lower respiratory tract infections. Crit Care 2010;14:R106.

22. Di Iorio R, Marinoni E, Scavo D, Letizia C, Cosmi EV. Adrenomedullin in pregnancy. Lancet 1997;349:328.

23. Fanaroff JM, Fanaroff AA. Blood pressure disorders in the neonate: hypotension and hypertension. Semin Fetal Neonatal Med 2006;11:174-81.

24. Barrington KJ. Low blood pressure in extremely preterm infants: does treatment affect outcome? Arch Dis Child Fetal Neonatal Ed 2011;96: F316-7.

25. Kamata S, Kamiyama M, Usui N, et al. Is adrenomedullin involved in the pathophysiology of persistent pulmonary hypertension of the newborn? Pediatr Surg Int 2004;20:24-6.

26. Nishikimi T, Matsuoka H, Shimada K, Matsuo H, Kangawa K. Production and clearance sites of two molecular forms of adrenomedullin in human plasma. Am J Hypertens 2000;13:1032-4. 
27. Marinoni E, Di Iorio R, Alò P, Villaccio B, Alberini A, Cosmi EV. Immunohistochemical localization of adrenomedullin in fetal and neonatal lung. Pediatr Res 1999;45:282-5.

28. Sugo S, Minamino N, Shoji H, et al. Interleukin-1, tumor necrosis factor and lipopolysaccharide additively stimulate production of adrenomedullin in vascular smooth muscle cells. Biochem Biophys Res Commun 1995;207:25-32.

29. Shoji H, Minamino N, Kangawa K, Matsuo H. Endotoxin markedly elevates plasma concentration and gene transcription of adrenomedullin in rat. Biochem Biophys Res Commun 1995;215:531-7.

30. Hirata Y, Mitaka C, Sato K, et al. Increased circulating adrenomedullin, a novel vasodilatory peptide, in sepsis. J Clin Endocrinol Metab 1996;81:1449-53.

31. Christ-Crain M, Morgenthaler NG, Struck J, Harbarth S, Bergmann A, Müller B. Mid-regional pro-adrenomedullin as a prognostic marker in sepsis: an observational study. Crit Care 2005;9:R816-24.

32. Hagag AA, Elmahdy HS, Ezzat AA. Prognostic value of plasma pro-adrenomedullin and antithrombin levels in neonatal sepsis. Indian Pediatr 2011;48:471-3.

33. Fowler DE, Yang S, Zhou M, Chaudry IH, Simms HH, Wang P. Adrenomedullin and adrenomedullin binding protein-1: their role in the septic response. J Surg Res 2003;109:175-81.

34. Trollmann R, Schoof E, Beinder E, Wenzel D, Rascher W, Dotsch J. Adrenomedullin gene expression in human placental tissue and leukocytes: a potential marker of severe tissue hypoxia in neonates with birth asphyxia. Eur J Endocrinol 2002;147:711-6.
35. Jensen RI, Carter AM, Skøtt O, Jensen BL. Adrenomedullin expression during hypoxia in fetal sheep. Acta Physiol Scand 2005;183:219-28.

36. Marinoni E, Pacioni K, Sambuchini A, Moscarini M, Letizia C, Di Iorio R Regulation by hypoxia of adrenomedullin output and expression in human trophoblast cells. Eur J Obstet Gynecol Reprod Biol 2011;154: 146-50.

37. Wellmann S, Benzing J, Cippà G, et al. High copeptin concentrations in umbilical cord blood after vaginal delivery and birth acidosis. J Clin Endocrinol Metab 2010;95:5091-6.

38. Benzing J, Wellmann S, Achini F, et al. Plasma copeptin in preterm infants: a highly sensitive marker of fetal and neonatal stress. J Clin Endocrinol Metab 2011;96:E982-5.

39. Caruhel P, Mazier C, Kunde J, Morgenthaler NG, Darbouret B. Homogeneous time-resolved fluoroimmunoassay for the measurement of midregional proadrenomedullin in plasma on the fully automated system B.R.A.H.M.S KRYPTOR. Clin Biochem 2009;42:725-8.

40. Benirschke K, Kaufmann P, Baergen RN. Pathology of the Human Placenta. 5th edn. Berlin: Springer, 2006.

41. Schlapbach LJ, Ersch J, Adams M, Bernet V, Bucher HU, Latal B. Impact of chorioamnionitis and preeclampsia on neurodevelopmental outcome in preterm infants below 32 weeks gestational age. Acta Paediatr 2010;99:1504-9.

42. Letzner J, Berger F, Schwabe S, et al. Plasma C-terminal pro-endothelin-1 and the natriuretic pro-peptides NT-proBNP and MR-proANP in very preterm infants with patent ductus arteriosus. Neonatology 2012;101:116-24. 